\section{Robust hybridization-based genotyping probes for HPV 6, 11,16 and 18 obtained via in vitro selection}

\author{
Ivan Brukner, 'Anne-Marie Larose, ${ }^{2}$ \\ Izabella Gorska-Flipot, ${ }^{3,4}$ \\ Maja Krajinovic,, ${ }^{1,5}$ Damian Labuda ${ }^{1,5}$ \\ 'Centre de Recherche, Hôpital Sainte- \\ Justine, Université de Montréal, \\ Montréal, Québec, Canada; \\ ${ }^{2}$ Gestion UNIVALOR, Limited Partnership, \\ Montreal, Quebec; \\ ${ }^{3}$ Centre de Recherche, CHUM, Hôpital \\ Hôtel-Dieu; \\ ${ }^{4}$ Département de Pathologie, Université \\ de Montréal; \\ ${ }^{5}$ Département de Pédiatrie, Université de \\ Montréal, Canada
}

\section{Abstract}

This paper describes the technical and analytical performance of a novel set of hybridization probes for the four GARDASIL ${ }^{\circledast}$ vaccinerelevant HPV types $(6,11,16$ and 18$)$. These probes are obtained through in vitro selection from a pool of random oligonucleotides, rather than the traditional "rational design" approach typically used as the initial step in assay development. The type-specific segment of the HPV genome was amplified using a $\mathrm{GP} 5 / 6+6^{+} \mathrm{PCR}$ protocol and 39 synthetic oligonucleotide templates derived from each of the HPV types, as PCR targets. The robust performance of the 4 selected hybridization probes was demonstrated by monitoring the preservation of the specificity and sensitivity of the typing assay over all 39 HPV types, using a different spectrum of HPV (genome equivalent: $10^{3}-10^{9}$ ) and human DNA concentrations (10-100 ng) as well as temperature and buffer composition variations. To the Authors' knowledge, this is a unique hybridization-based multiplex typing assay. It performs at ambient temperatures, does not require the strict temperature control of hybridization conditions, and is functional with a number of different non-denaturing buffers, thereby offering downstream compatibility with a variety of detection methods. Studies aimed at demonstrating clinical performance are needed to validate the applicability of this strategy.

\section{Introduction}

The HPV vaccination era calls for new stan- dards for HPV screening programs. ${ }^{1.3}$ National public health institutions require the monitoring of vaccine efficacy and HPV typing has become clinically and epidemiologically relevant. Two HPV diagnostic assays have been approved by the US Food and Drug Administration (Hybrid Capture 2, HC-2, Qiagen and Cervista HPV 16/18 genotyping tests, Hologic, Beford, Mass., USA), while Linear Array HPV genotyping test (Roche) and INNO-LiPA HPV Genotyping v2 (Innogenetics) assay are registered for use in the European Union. They are followed by a spectrum of other assays in late development and early validation phases. ${ }^{4-6}$ These assays demand sophisticated instrumentation, significant material cost outlays and technically-skilled personnel. Concordance among various assays under certain conditions (e.g. mixed infections) still needs to be technically resolved. ${ }^{7,8}$ The next generation of HPV typing assays (bead-based platforms) are in the developmental phase (for research purposes only) and although they have a higher throughput capacity and elegant design, their overall assay cost represents a heavy burden for national health care screening programs. ${ }^{9-15}$ Of additional concern is that most of the world's population does not have access to the equipment required to perform current HPV typing procedures. ${ }^{16}$ Encouraging efforts have been made by PATH-QIAGEN to introduce screening assays to developing countries. ${ }^{17}$ However, a robust typing assay still does not exist. At the heart of this problem is a dominant technological and scientific hurdle imposed by the current limited understanding of the specificity of nucleic acid interactions.

A recently described technique ${ }^{18-20}$ was applied to bring HPV typing one step closer to robust performance. The technique uses in vitro selection procedures to generate oligonucleotide probes with type-specific resolution. Hybridization probes, empirically obtained through selection, are used to build a novel hybridization assay that is applicable to a number of different platforms. The assay uses welldescribed $\mathrm{GP}^{+} / 6^{+} \mathrm{HPV} \mathrm{PCR}^{21,22}$ with minor modifications. ${ }^{19}$ After amplicon conversion to the single stranded form, hybridization with type-specific probes is performed. Assay performance is further analytically tested under varying conditions: a different HPV copy number within the context of human DNA and a range of temperatures under which robust hybridization and washing may typically be performed. To the Authors' knowledge, this is a unique hybridization-based multiplex typing assay that does not require temperature-controlled hybridization conditions. Due to the inherent features of selected probes, the hybridization assay is operative within different (non-denaturing) buffers. That feature might provide downstream compatibility with a number of different detection techniques.
Correspondence: Ivan Brukner and Anne-Marie Larose, Department of Medical Diagnostics, SMBD-Jewish General Hospital, Suite/Room D133, 3755 Cote-Sainte-Catherine Road, Montreal, Quebec, Canada. Email: ibrukner@hotmail.com

Key words: HPV assay, hybridization, cervical cancer, HPV typing, multiplex assay.

Acknowledgments: we would like to thank Dr. Larry Gold (SomaLogic, USA), Dr. André Dascal (McGill, JGH, Montreal), Drs. Markus Schmitt and Michael Pawlita (Heidelberg, Germany), Irina Nazarenko (Qiagen, USA), Kerry Gunning (IDT, USA) and Alex Ferenczy (McGill, JGH, Montreal) for encouragement and useful advice. This work was supported by the Canadian Institutes of Health Research (POP-CIHR) and the Research Center of the CHU Sainte-Justine. MK is a research scholar of the Fonds de la Recherché en Santé du Québec.

Received for publication: 17 November 2009

Revision received: 1 March 2010.

Accepted for publication: 3 March 2010.

This work is licensed under a Creative Commons Attribution 3.0 License (by-nc 3.0).

(C) Copyright I. Brukner et al., 2010

Licensee PAGEPress, Italy

Journal of Nucleic Acids Investigation 2010; 1:e3 doi:10.4081/jnai.2010.e3

In this work we hypostatize that: (a) selected probes ${ }^{19}$ do have robust performance (tolerate $8-10^{\circ} \mathrm{C}$ operative range) and preserve analytical sensitivity of 1,000 genome equivalents of HPV in the context of 10,000 (or less) of human genome equivalents; and (b) selected probes preserved specificity over the same conditions as in (a) for a specific target (either 6 , or 11 , or 16 , or 18 ) and did not show any cross-hybridization with any of the remainder 38 HPV types, for which counterselection was performed.

\section{Materials and Methods}

\section{Oligonucleotides}

The oligonucleotides were synthesized by Integrated DNA Technologies (Coralville, Iowa, USA). Targets were 139-148 nucleotide-long, type-specific segments (GP5 ${ }^{+}$strands) of $\mathrm{GP}^{+} / 6^{+}$amplicons, located between positions 6624 and 6765 of the HPV16 sequence (accession number K02718). The 5' block TTCGACAGGAGGCTCACAACAGGC and 3' block TGGGAGACAAGAATA AACGCTCAA were used to "block" the anchoring segments of the probes. The forward $\mathrm{GP}^{+}$primer has been previously described ${ }^{20}$ and a mixture of 4 oligonucleotides was used as a reverse $\mathrm{GP} 6+$ primer: 
GAAAAATAAACT G TAAAT CATAT T C, GAAAAATAAAC T G TAAAT CATAC T C, GAAAAATAAACTGTAAATCAAATTC and GAAAAATAAACTGTAAATCAAACTC. ${ }^{19}$ The universal HPV probe (UP) sequence was: GAAAAATAAACTGTAAATCATATTCCTCCACATGTCTTATATATTCCTTAAA. All type-specific probes were obtained using an in vitro selection procedure (Figure 1). The selected probes used in this assay were: i) for HPV 6: 5'GCCTGTTGTGAGCCTCCTGTCGAAGGAAGATGTAGGTAGGGATCGA TTGAGCGTTTATTCTTGTCTCCCA3'; ii) for HPV11: 5'GCCTGTTGTGAGCCTCCTGTCGAATTAGTGTATGTAGCATGCGACA TTGAGCGTTTATTCTTGTCTCCCA3'; iii) for HPV16: 5'GCCTGTTGTGAGCCTCCTGTCGAA GATCGGGAAGTAGATATGGCGC TTGAGCGTTTATTCTTGTCTCCCA3'; iv) for HPV 18: 5'GCCTGTTGTGAGCCTCCTGTCGAA GAGACAGGTAGAAGGGCCCAGG TTGAGCGTTTATTCTTGTCT CCCA3'.

\section{DNA extraction}

Human DNA used for spiking experiments was extracted from blood (QIAamp DNA tubes, Qiagen, Mississauga, Ont., Canada), while the concentration was measured using NanoDrop (NanoDrop ND-1000, Wilmington, Del., USA) and DNA integrity was validated on $0.7 \%$ agarose gel.

\section{PCR}

The $50 \mu \mathrm{L}$-volume PCR was performed as originally suggested ${ }^{20}$ with minor modifications including shortening the elongation and denaturation time to $20 \mathrm{sec}$. This modification was applied to minimize non-specific amplification, which was present in the original protocol, ${ }^{20}$ especially in the cases where the copy number of HPV targets was below 1,000 HPV viral copies in the context of 1,000 or more genome copies of human DNA. PCR yield was monitored by loading a $10 \mu \mathrm{L}$ reaction mix on 1.5\% agarose gel and EtBr staining.

\section{Conversion of PCR product to sin- gle-stranded (ss) DNA and labeling}

The rest of the PCR mix $(40 \mu \mathrm{L})$ was digested for $15 \mathrm{~min}$ at $37^{\circ} \mathrm{C}$ with Exonuclease I (New England Biolabs Ltd., Pickering, Ont., Canada) to remove excess primers following enzyme inactivation $\left(20 \mathrm{~min}, 85^{\circ} \mathrm{C}\right)$. Lambda exonuclease (New England Biolabs Ltd., Pickering, Canada) was used $\left(15 \mathrm{~min}, 37^{\circ} \mathrm{C}\right)$ to remove a GP6 ${ }^{+}$PCR strand, followed by an enzyme inactivation step $\left(20 \mathrm{~min}, 85^{\circ} \mathrm{C}\right)$. The sample was passed through a Sephadex G-25 (GE Healthcare Life Science, Baie d'Urfé, Quebec, Canada) for buffer exchange reaction compatibility with a downstream T4 polynucleotide kinase (Invitrogen, Burlington, Ont., Canada) labeling step.

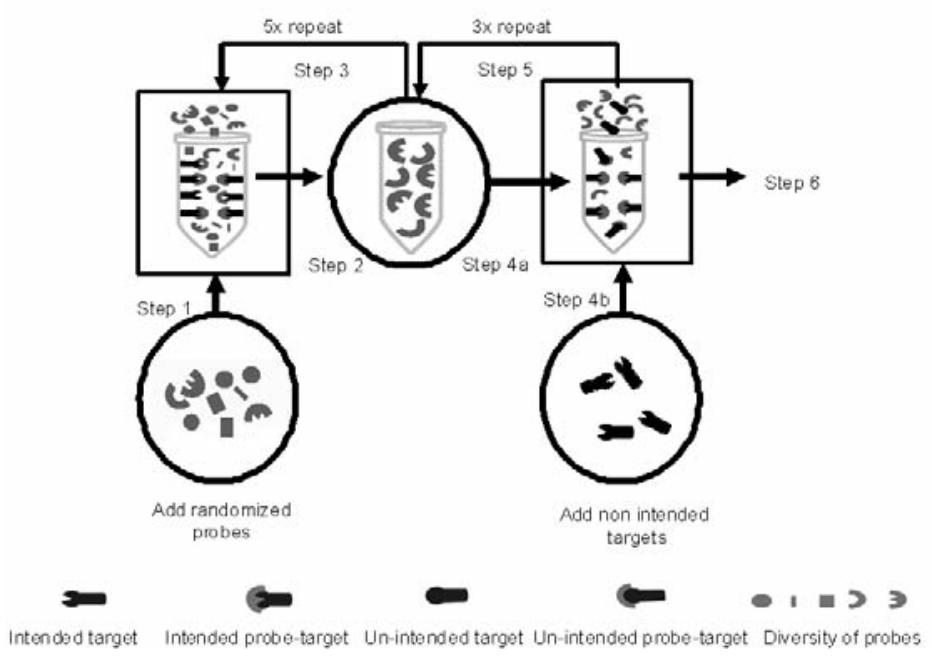

Figure 1. Iterative hybridizations. Methodology used for the selection of probes (modified from ${ }^{18}$ ). The technique involves five forward hybridizations (steps 1-3) to generate pools of probes with significant affinity, but not necessarily specificity, for the target. Specificity is then achieved during subtractive hybridization steps (steps 4-6) where only probes having a differential diagnostic performance are retained. Iterative hybridizations, cloning, sequencing and testing of the performance of selected probes can all be fully automated. Intended and non-intended targets, probes and complexes between them are shown in the legend (see below). Note that the intended targets are attached to the solid support, while non-intended targets are free in solution and present only in subtractive hybridization steps.

\section{Membrane}

Streptavidine-coated Promega membrane (SAM, Biotin Capture Membrane, Medison, Wisc., USA) was used in the following manner. One $\mu \mathrm{L}$ of $100 \mathrm{pmol} / \mu \mathrm{L}$ of type-specific 5 ' biotinilated oligonucleotide probes was manually spotted (HPV6, HPV11, HPV 16 and HPV 18) on the surface of a $3 \times 2 \mathrm{~cm}$ membrane (see Figure 2 for spotting schematic). The $0.3 \mathrm{pmol}$ of the HPV universal probe (UP) was spotted on the membrane and dried at ambient temperature for $5 \mathrm{~min}$. The membrane was washed in $\mathrm{H}_{2} \mathrm{O}$ for 1-2 min and pre-hybridized in $2 \mathrm{~mL}$ hybridization buffer: SSPE $(150 \mathrm{mM} \mathrm{NaCl}, 10$ $\mathrm{mM} \mathrm{NaH}_{2} \mathrm{PO}_{4}, 1.1$ mM EDTA, pH 7.4), containing $1 \% \quad$ SDS and $200 \mathrm{mg} / \mathrm{mL}$ heparin (hybridization oven, Model 400, Robbins Scientific) for $1-12 \mathrm{~h}$ at $55^{\circ} \mathrm{C}$. The membrane was either stored at room temperature (for as long as four days) or immediately used for hybridization assay.

\section{Labeling}

Labeling (SA, specific activity of $10^{5}-10^{6}$ $\mathrm{cpm} / \mathrm{pmol}$ ) of 2-20 pmol of single-stranded PCR product and/or an oligonucleotide was performed using $5 \mu \mathrm{L}$ of [ $\gamma$-32P] ATP (6000 $\mathrm{Ci} / \mathrm{mmol}$ ) and $1 \mu \mathrm{L}$ of $\mathrm{T} 4$ polynucleotide kinase, following the manufacturer's recommendations (Invitrogen, Burlington, Ont., Canada).

\section{Hybridization}

Hybridization was carried out in the hybridization buffer for 1-12 h, at ambient tem- peratures $\left(22 \pm 2^{\circ} \mathrm{C}\right)$. To analyze the effect of temperature variation, hybridization was also carried out at fixed temperatures (Figure 3). The membrane was washed with hybridization buffer containing $0.1 \%$ SDS for $10 \mathrm{~min}$ at ambient temperature and either exposed overnight at $-80^{\circ} \mathrm{C}$ with intensifying screens or exposed in Cyclone Storage Phosphore Screen (Perkin Elmer Life and Analytical Sciences, Shelton, Conn., USA) for 10-30 min and read by Cyclone software (OptiQuant, version 4.00, Packard Instrument Co., Boston, Mass., USA).

\section{Results}

The performance of a new generation of HPV-type specific hybridization probes is presented. They were obtained using in vitro selection as opposed to rational and computerassisted in silico design and optimization. A set of such probes for 39 HPV types has been previously obtained..$^{19}$ Briefly, selection from the random pool of probes was composed of 5 preparative hybridization steps (Figure 1, Steps 1 to 3) using the intended type-specific HPV target, followed by 3 subtractive hybridization steps (Figure 1, Steps 4 to 6). During subtractive steps, the remaining 38 non-intended targets were added into the hybridization mix. The selected probes were cloned, sequenced, chemically synthesized and tested for their specificity. ${ }^{19}$ Here, the focus was on 4 vaccinerelevant HPV-types. The assay format, including the 4 corresponding probes, was dictated 
by its clinical relevance and expected costeffectiveness.

The experiments were performed to test the limits of the specificity, sensitivity and robustness of the assay. First, the specificity of hybridization probes was tested under conditions where the concentration of targets was about 10 times higher than that of the typical PCR yield. All probes were hybridized in the presence of 20 pmol of all 39 HPV type-specific single-stranded oligonucleotides, identical to a $\mathrm{GP}^{+}$primed single strand of double stranded PCR. Our typical PCR yield does not exceed $1 \mu \mathrm{g}$ of product (from $50 \mu \mathrm{L}$ reaction mixture). Therefore, maximal number of post-PCR molecules is lower then tens of picomols. Tested probes were hybridized with 20 pmols of each of the 39 type-specific amplicons (synthetic oligonucleotides) and neither (except intended targets) gave any detectable signal. The fact that there was no observed cross-hybridization even in an extremely high concentration of HPV amplicons, indicates high specificity of the selected probes.

Second, the sensitivity of the assay was tested using PCR amplified targets in the context of 10 and $100 \mathrm{ng}$ of human DNA (Figure 2) approximating clinical samples in the range of $10^{3}-10^{4}$ human genome equivalents. While estimating assay sensitivity, we used terminology of minimal numbers of required genome equivalents per reaction mix. This term would be equivalent to the viral copy numbers per assay, without refereeing to the assay volume units. Viral loads reflecting 100 and 10,000 copy numbers of HPV were analyzed in the context of variable genome-equivalents of human DNA (Figure 2A-C). For example, samples having 10:1 and 1:1 ratios between HPV and human genome equivalents produced clear patterns (Figure $2 \mathrm{~A}$ and B). However, a 1:10 ratio between HPV and human genome copies produced noise in the hybridization patterns (Figure 2C). The 100 copies of HPV in the context of 1,000 human genome equivalents were not amplified well enough to be detected. These results reflect the present limits of assay sensitivity, which are related to the present limits of PCR sensitivity.

Clinical samples, which were previously genotyped by RFLP analysis using the MY 09/MY 11 primer set, were analyzed (Figure 2 D). RFLP vs. hybridization assay produced the same typing data when single infections were considered. When multiple infections were simulated (equimolar quantities of 2-4 targets), the PCR amplified oligonucleotide targets produced type-specific signals of uneven intensity (data not shown). However, when hybridization was carried out without intermediate PCR amplification, the hybridization patterns were proportional to the concentration of input multiple target templates (again equimolar quantities of 2-4 targets). A similar

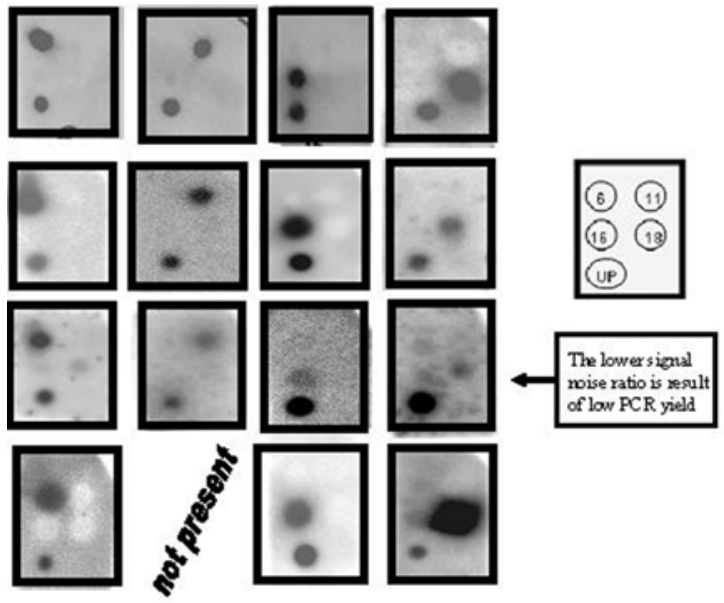

Figure 2. Assay specificity and sensitivity. Autoradio graphy results of HPV typing assay using reconstructed and clinical samples. Reconstructed samples (see Materials and Methods): (A) 10,000 copies of HPV in the context of $10 \mathrm{ng}$ of human DNA ( 3300 haploid equivalents); (B) 1,000 copies of HPV and 10 ng human DNA; (C) 1,000 copies of HPV and $100 \mathrm{ng}$ of human DNA. (D) An example of hybridization observed in clinical samples. UP - Universal HPV probe.

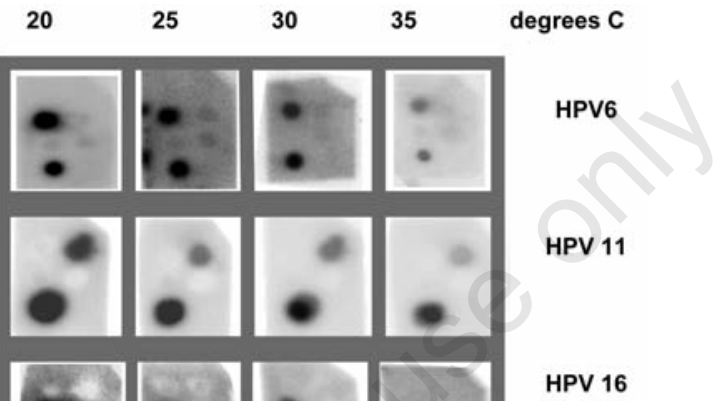

observation has recently been noted with clinical samples: mixed infections produced highly disconcordant typing results among a variety of assays. ${ }^{8}$

To evaluate the robustness of the assay, hybridization was carried out at different temperatures in the range of $20-35^{\circ} \mathrm{C}$ (Figure 3 ). The hybridization buffer was changed from the one previously used ${ }^{19}$ for the selection of HPV typing probes (10 mM Tris-HCl, pH 7.5, $10 \mathrm{mM}$ $\mathrm{MgCl}_{2}$ and $50 \mathrm{mM} \mathrm{NaCl}$ ) to the one described in the Methods section in order to better fit the membrane-based hybridization requirements.

\section{Discussion}

HPV typing using multiplex hybridization assays is facing two distinct technical issues. The first one concerns cross-hybridization among non-intended targets. As shown by the present evaluation of the sensitivity, specificity and robustness of our HPV typing assay to detect 4 GARDASIL ${ }^{\circledR}$-relevant HPV types $(6,11$, 16 and 18), this issue was successfully resolved. The second technical issue is more general: an amplification bias caused by the use of a variety of different PCR-based "broadrange" primer sets. The roots of both problems, stemmed from an insufficient knowledge of sequence-dependent interactions among nucleic acids. The second technical problem caused significant non-concordance among different typing assays, when reporting double or multiple HPV infections, "probably due to the differences in the affinities of the primer sets for the different HPV types". Therefore, clinical validations of a range of primer designs $^{14}$ and improved amplification procedures or pre-PCR sample enrichments ${ }^{12}$ are necessary.

The 4 selected probes $(6,11,16$ and 18$)$ were tested against 39 different HPV types under defined conditions (presented as hypotheses (a); see above). Only intended target sequences showed hybridization signal, while the rest of non-intended targets did not show any detectable cross-hybridization. The signal had a minimum 10 times stronger $\mathrm{P}^{32}$ number of counts per minutes than background, over the same membrane surface. The visual presentation of autoradiography of membranes (Figures 2 and 3) illustrates typical signalnoise ratio. Non-intended targets (all 38) did not show any cross-hybridization with 4 select- 
ed and spotted probes, which is in agreement with our fluorescence assay presented in a previous work. ${ }^{19}$ The 100 copies of HPV in the context of 1,000 GE of human DNA was difficult to detected (signal - noise ration lower than 10), while 1,000 HPV copies were clearly detected for all types. The current sensitivity of the assay described herein is comparable with the lower limits of detection of FDA-approved HC-2 (1,000 copies of HPV). However, one should note that sensitivity can be increased by: i) changing the original $\mathrm{GP}^{+} / 6^{+}$PCR conditions; ii) using newer primer sets described in the literature ${ }^{14}$ re-designing new primer sets.

This assay was tested at 4 hybridization temperatures $\left(20^{\circ} \mathrm{C}, 25^{\circ} \mathrm{C}, 30^{\circ} \mathrm{C}\right.$ and $\left.35^{\circ} \mathrm{C}\right)$ (Figure 3). While hybridization intensity increased with decreasing temperature, the assay specificity was preserved over this range of temperature conditions. This feature is advantageous when compared with other commercial HPV typing assays where small variations of $1-2^{\circ} \mathrm{C}$ lead to cross-hybridization or loss of sensitivity. ${ }^{23,24}$

The assay performance was well tolerated using 2 different hybridization buffers. This inherent feature of selected probes is important, considering different buffer requirements of enzyme-driven colorimetric reactions that could eventually be applied here to detect a specific hybridization event.

The attractive use of this type of assay in clinical practice would be isothermal amplification (Biohelix, US) combined with chromatographic hybridization detection (ChemBio Diagnostics), offering a hands off, instruments off, simple, multiplex, point-ofcare diagnostic test.

It is clear that the current form of the assay would need to address a combination of add tional optimization factors to achieve required sensitivity, while switching from $\mathrm{P}^{32}$ to colorimetric, or fluoromoetric detection.

The technique of the in vitro selection of oligonucleotides of diagnostic and therapeutic value was introduced in 1990 by Tuerk, Gold, Ellington and Szostak. ${ }^{25,26}$ The demonstrated robustness of hybridization probes under various "environmental conditions" represents an unexpected result. It seems that selection-captured molecular determinants in probe-target complexes are only those that are consistently preserved under imposed conditions of selection. This feature is very useful and should encourage the development of a fast and automatic generation of diagnostic probes.

Based on the overall results, it can be concluded that the in vitro selection of hybridization probes is a valuable technological choice for engineering multiplex robust point-of-care diagnostic assays. Further studies aimed at avoiding amplification bias are needed to illustrate the universal clinical applicability of these types of molecular detection assay.

\section{References}

1. Kiviat NB, Hawes SE and Feng Q. Screening for cervical cancer in the era of the HPV vaccine - the urgent need for both new screening guidelines and new biomarkers. J Natl Cancer Inst 2008;100:290-1.

2. Myers E, Huh WK, Wright JD, Smith JS. The current and future role of screening in the era of HPV vaccination. Gynecol Oncol 2008; 109:S31-9.

3. Rogoza RM, Ferko N, Bentley J, et al. Optimization of primary and secondary cervical cancer prevention strategies in an era of cervical cancer vaccination: a multi-regional health economic analysis. Vaccine 2008:26; F46-58.

4. Keegan H, McInerney J, Pilkington L, et al. Comparison of HPV detection technologies: Hybrid capture 2, PreTect HPV-Proofer and analysis of HPV DNA viral load in HPV16, HPV18 and HPV33 E6/E7 mRNA positive specimens. J Virol Methods 2009;155:61-6.

5. van Ham MA, Bakkers JM, Harbers GK, et al. Comparison of two commercial assays for detection of human papillomavirus (HPV) in cervical scrape specimens: validation of the Roche AMPLICOR HPV test as a means to screen for HPV genotypes associated with a higher risk of cervical disorders. J Clin Microbiol 2005;43:2662-7.

6. van Hamont D, van Ham MA, Bakkers JM, et al. Evaluation of the SPF10-INNO LiPA human papillomavirus (HPV) genotyping test and the Roche linear array HPV genotyping test. J Clin Microbiol 2006;44: 3122-9.

7. Quint WG, Pagliusi SR, Lelie N, et al. Results of the first world health organization international collaborative study of detection of human papillomavirus DNA. J Clin Microbiol 2006;44:571-9.

8. Sabol I, Salakova M, Smahelova J, et al. Evaluation of different techniques for identification of human papillomavirus types of low prevalence. J Clin Microbiol 2008;46:1606-13.

9. Dalstein V, Merlin S, Bali C, et al. Analytical evaluation of the PapilloCheck test, a new commercial DNA chip for detection and genotyping of human papillomavirus. J Virol Methods 2009;156:77-83.

10. Jiang HL, Zhu HH, Zhou LF, et al Genotyping of human papillomavirus in cervical lesions by L1 consensus PCR and the Luminex xMAP system. J Med Microbiol 2006;55:715-20.

11. Klaassen CH, Prinsen CF, de Valk HA, et al. DNA microarray format for detection and subtyping of human papillomavirus. J Clin Microbiol 2004;42:2152-60.

12. Nazarenko I, Kobayashi L, Giles J, et al. A novel method of HPV genotyping using Hybrid Capture sample preparation method combined with GP5+/6+ PCR and multiplex detection on Luminex XMAP. J Virol Methods
2008;154:76-81.

13. Schmitt M, Bravo IG, Snijders, PJ, et al. Beadbased multiplex genotyping of human papillomaviruses. J Clin Microbiol 2006;44:504-12.

14. Schmitt M, Dondog B, Waterboer T, Pawlita M. Homogeneous amplification of genital human alpha papillomaviruses by PCR using novel broad-spectrum GP5+ and GP6+ primers. J Clin Microbiol 2008;46:1050-9.

15. Wallace J, Woda BA, Pihan G. Facile, comprehensive, high-throughput genotyping of human genital papillomaviruses using spectrally addressable liquid bead microarrays. J Mol Diagn 2005;7:72-80.

16. Tsu V and Levi CE. Making the case for cervical cancer prevention: what about equity? Reprod Health Matters 2008;16: 104-12.

17. Qiao YL, Sellors JW, Eder PS, et al. A new HPV-DNA test for cervical-cancer screening in developing regions: a cross-sectional study of clinical accuracy in rural China. Lancet Oncol 2008;9:929-36.

18. Brukner I, El-Ramahi R, Gorska-Flipot I, et al. An in vitro selection scheme for oligonucleotide probes to discriminate between closely related DNA sequences. Nucleic Acids Res 2007;35:e66.

19. Brukner I, El-Ramahi R, Sawicki J, et al. Hybridization assay performed at ambient temperature for typing high-risk human papillomaviruses. J Clin Virol 2007;39:113-8.

20. Brukner I, Krajinovic M, Dascal A, Labuda D. A protocol for the in vitro selection of specific oligonucleotide probes for high-resolution DNA typing. Nat Protoc 2007;2:2807-14.

21. de Roda Husman AM, Walboomers JM, van den Brule AJ, et al. The use of general primers GP5 and GP6 elongated at their $3^{\prime}$ ends with adjacent highly conserved sequences improves human papillomavirus detection by PCR. J Gen Virol 1995;76:105762.

22. Jacobs MV, Snijders PJ, van den Brule AJ, et al. A general primer GP5+/GP6(+)-mediated PCR-enzyme immunoassay method for rapid detection of 14 high-risk and 6 low-risk human papillomavirus genotypes in cervical scrapings. J Clin Microbiol 1997;35:791-5.

23. Steinau M, Swan DC, Unger ER. Type-specific reproducibility of the Roche linear array HPV genotyping test. J Clin Virol 2008;42:4124.

24. Stevens MP, Garland SM, Tabrizi SN. Human papillomavirus genotyping using a modified linear array detection protocol. J Virol Methods 2006;135:124-6.

25. Ellington $A D$, Szostak JW. In vitro selection of RNA molecules that bind specific ligands. Nature 1990;346;818-22.

26. Tuerk C and Gold, L. Systematic evolution of ligands by exponential enrichment: RNA ligands to bacteriophage T4 DNA polymerase. Science 1990;249: 505-10. 